\title{
Stage 0 Fallopian Tube Cancer AJCC v7
}

National Cancer Institute

\section{Source}

National Cancer Institute. Stage O Fallopian Tube Cancer A/CC v7. NCI Thesaurus. Code C40105.

Stage 0 includes: T is, N0, M0. Tis: Carcinoma in situ (limited to tubal mucosa). N0: No regional lymph node metastasis. MO: No distant metastasis. FIGO no longer includes stage 0. (AJCC 7th ed.) 